\title{
Application of Computer Image Processing Technique in Medical Science
}

\author{
Li Yan-Ming1 a , SongWeiZhong2b \\ 1 The Student Physical Health Institute of Henan University (Huaihe Hospital )Kaifeng 475001 \\ China \\ ${ }^{2}$ College of Pharmacy,Henan University Kaifeng 475001 China \\ aemail:15203789966@163.com; bemail:13663788266@163.com
}

Key words: computer technique, medical image, image processing technique

\begin{abstract}
The modern medicine has closer relationship with the support from the medical image information, medical facility or system. In the medical image processing and medical facility, the ultrasonic imaging, CT, magnetic resonance, surgery, and traditional Chinese medicine diagnosis are closely related to the computer image processing technique.
\end{abstract}

\section{Introduction}

With the constant development of the computer technology, the computer image processing technique has played a vital role in the medical science. For instance, with regard to the computer-aided diagnosis, it can help the doctors to make precise quantitative calculation and improve the diagnosis accuracy, which greatly reduces the probability of misdiagnosis. Besides, in the surgery, it can utilize the numbers to affect the technology and then obtain 2D cross-sectional images of the sick body, and then adopts the computer software for the analysis and processing, and finally carries out pre-operation design and post-operation treatment. Thus, it can be seen that, to calculate the geometry image and position of the sick body put forward by the image processing technique improves the accuracy and safety of the doctors' diagnosis.

At present, the medical image processing techniques applied by China are mainly medical image fusion technique, medical image segmentation technique, medical image registration technique and 3D image reconstruction technique. The medical image processing technique not only has the advantages possessed by the verbal description, but also has the characteristics of perceptual intuition and expression, which can improve our medical diagnosis quality to a great extent. Therefore, we should make effort to push forward the development of the computer image processing technique in the medical science domain, which not only can improve our diagnosis quality, but also can lay sold foundation for our future medical development.

\section{Categories of the Medical Image}

With the development of the computer technology and medical science, the medical image information plays a more and more important role in the clinical diagnosis. At present, the medical images required for the medical research and clinical diagnosis are various, such as B ultrasonic image, MRI image, CT image, PET image, SPECT image, digital X ray (DR) machine, $X$ ray image, various electronic endoscopy images, case section and cell image under the microscope, etc. to apply the computer technology to process these images not only can improve the clinical diagnosis level, but also can provide necessary support for the medical training, research and teaching, computer-aided clinical surgery, etc. 


\section{The Principle of Image Technology}

Image processing technology has made development for a long period. Especially with the rapid development of computer technology, it also has got rapid development. In the broad sense, the image technology is the general term of a variety of image-related technologies. At present, people mainly study the digital image, and the main application is the computer graphics technology. This includes that use computers and other electronic devices to complete a series of work. For example, the image collecting, acquisition, coding, storage and transportation, and the image synthesis and generation, display and output of the image, image conversion, enhancement, recovery (restoration) and reconstruction, image segmentation, target detection, expression and description, the feature extraction and measurement, image sequences correction, 3-D scene reconstruction recovery, establishing image database, index, and extraction, image classification, representation and identification, model image establishing and matching, and the interpretation and understanding on image and scene, decision and action plans based on their judgment and so on. 1980s and 1990s, the image processing technology can not only be carried out in a two-dimensional spatial processing and application, but also it can be carried out in three-dimensional spatial application and it has been booming development, such as three-dimensional animation, film special effects technology and engineering graphics design, etc. . We can say that image technology has been involved in all aspects of human life and social development. The complex processing results of video image technology is to put everything in static image or dynamic video form to show in front of people, so we do not need to have a very deep understanding on the encode and imaging process of the image processing technology. In medical research service, we mainly use hardware and software systems associated with the image processing technology. We make the video image results into different forms of application and development, with the most effective ways and means to improve the medical level.

\section{Content of the Medical Image Processing Technology}

In the medical image processing, the computer plays a vital role. The generalized image processing technique includes: image obtaining, image storing, image transmitting, image processing and image outputting. The above processing requires the computer technique. The narrow image processing mainly studies the algorithm that can be realized by the computer, including the following aspects. (1) Geometric processing: including changing the image size, rotating and moving the image, etc. (2) Algorithm and logic budget: including the adding, subtracting, multiplying and dividing of the image, and-or-not operation, etc. (3) Image digitalization: transform the image with the analog form into the digital image. (4) Image transformation: to provide convenience for the following operation, change the representative domain of the image and present the data, such as contrast enhancement, smoothness, calibration, etc. (6) Image restoration: restore the distorted image and make it get closer to the original image, such as restoration method in the frequency domain, maximum entropy restoration, motion blurring restoration, etc. (7) Image compression: to provide convenience for the image transmitting and stroring, transform a big data file into a small connatural file, such as adaptive compression, compression based on the artificial neural network and wavelet technology, etc. (8) Image segmentation: segment the interesting part from the image, to lay foundation for the following image analysis and comprehension, such as boundary detection, region detection, etc, which can be referred to the references [2], etc. (9) Image presentation and description: make further representation and description towards the segmented image, so as to become more suitable for the further processing of the computer, such as color extraction, texture extraction, regional collection feature, etc. (10) Image classification identification: classify and identify the image according to the extracted features, such as artificial neural network, support vector machine, fuzzy recognition, etc. (11) Image reconstruction: reconstruct the the technique of the 2D or 3D image of the profile, such as projective reconstruction, 3D reconstruction technology, etc. In general, the image processing 
refers to the narrow image processing.

\section{Application of the Computer Image Processing Technique in the Medical Science}

5.1 Application the image processing technique in the ultrasonic medical imaging

There are many image processing methods in the ultrasonic medical imaging. The main ones are image smoothing processing, image false color processing, image texture analysis, image segmentation, image sharpening processing, image enhancement processing, etc. [3]. In the B-mode ultrasound image, there is inevitably noise. It will have impact on a certain pixel or a certain picture. Therefore, it is necessary to smooth the image, remove the noise, and prepare for the following processing of the image. To enable the B-mode ultrasound doctor to better identify the image information, it is feasible to use different colors to present the different gray levels in the image, and reach the effect of the image enhancement, and identify the pixel with the relatively small gray difference. Such image that is composed by adopting the color different to replace the gray level difference is named false color image. There is particle texture in the B-mode ultrasound image, which is mainly caused by the following two situations. The first one is the stripe of the B-mode ultrasound image, and it is useless information. The second one is caused by the organization structure of the people being tested, and it is useful information. The image tissue particle of the normal and lesion organs is differently distributed. Namely, the texture is different. Therefore, analyze the texture of the B-mode ultrasound image, and then judge the state of the illness. The image segmentation is to segment the lesion region, so as to provide convenience to measure the size, volume, etc, which can provide necessary data for the diagnosis. In addition, the image sharpening processing, image enhancement and other computer technique should also be adopted for processing the B-mode ultrasound image.

5.2 Application of image processing technique in CT and MRI

The essence of CT is cross-sectional image technique for imagining and data processing by virtue of the computer. Although X-ray perspective enable people to understand the internal structure of the human body, the resolution ratio only can be fundamentally solved after CT eliminates the interference of the scattered rays and superimposed image through the computer and makes quantitative analysis on the system matrix. That the computer finishes the image denoising, image enhancement, image reconstruction and other related tasks in the CT system cannot be imagined without the development of the computer technique and CT device [4]. In the magnetic resonance, the image processing technique includes image denoising, restoration, 3D construction, etc. It cannot be separated from the support of the computer image processing technique.

5.3 Application of the image processing technique in the image guided surgery (IGS)

The Surgical navigation is one of the minimally invasive surgeries (MIS) with rapid development in recent 20 years. IGS system uses the medical image and computer image processing technique to carry out 3D reconstruction and visualization on the multimodality image data of the patients before the operation, gain the 3D model, formulate reasonable and quantitative operation plan, and carry out the pre-operation simulation; in the operation, utilize the 3D spatial orientation system to proceed with the registration or rectification of the image and patient physical space, and reflect the patients' actual position and the real-time spatial position of the operation instrument to the 3D image space of the patient, and collect and present the position of the operation instrument in the space at real time. Through observing the relative position relationship between the operation instrument and lesion part in the 3D image, the doctor performs accurate surgery on the patient [5]. It combines the image and graphics processing, spatial stereoscopic positioning, precision machinery and surgery. The medical image automatic processing algorithm, such as image segmentation, filtering, and feature extraction algorithm, plays a vital role in IGS.

5.4 Application of image processing technique in the traditional Chinese medicine tongue picture diagnosis system

The computer image processing technique plays a vital role in the comprehensive quantitative research of the tongue picture, and also one of the modern development directions of the tongue diagnosis. The traditional Chinese tongue image diagnosis system adopts the colorimetry, modern 
optical technology, image processing technique, computer hardware technique, etc. The image processing technique is one of the key techniques. In this system, it is necessary to pre-process the tongue image, including denoising, image segmentation, and other operations. Establish color model, confirm the definition domain related to the tongue image according to the fuzzy mathematic theory, and carry out the feature extraction, and texture analysis. All of them are computer image processing techniques.

\section{References}

[1] Pan Liqing. Application of Computer Technology in Medical Instrument [M]. Beijing, China Institute of Traditional Chinese Medicine, 2008: 69-89.

[2] Zhang Guiying, Zhang Xianjie. Research on Medical Image Segmentation Technique [J]. Medical Information, 2011, 24(1): 533-535.

[3] SIMI Realit y M otion Sys tems GmbH. SIMI M ot ion UserManual[ CP/ DK] . 2002.

[4] Li Yibing, Yu Dakun. Application of Computer in Ultrasound Medicine Image Processing [J]. Medical Information, 2005, 18(9): 1035-1036.

[5] LiuXiao-Wei. Image processing technology [ M ] . BeiJing: QingHua University Press, 2004. 